\title{
Motivational Responses to Natural and Drug Rewards in Rats with Neonatal Ventral Hippocampal Lesions: An Animal Model of Dual Diagnosis Schizophrenia
}

\author{
R. Andrew Chambers, M.D., and David W. Self, Ph.D.
}

The high prevalence of substance use disorders in schizophrenia relative to the general population and other psychiatric diagnoses could result from developmental neuropathology in hippocampal and cortical structures that underlie schizophrenia. In this study, we tested the effects of neonatal ventral hippocampal lesions on instrumental behavior reinforced by sucrose pellets and intravenous cocaine injections. Lesioned rats acquired sucrose selfadministration faster than sham-lesioned rats, but rates of extinction were not altered. Lesioned rats also responded at higher rates during acquisition of cocaine self-administration, and tended to acquire self-administration faster. Higher response rates reflected perseveration of responding during the post-injection "time-out" periods, and a greater incidence of binge-like cocaine intake, which persisted even after cocaine self-administration stabilized. In contrast to sucrose, extinction from cocaine self-administration was prolonged in lesioned rats, and reinstatement of cocaine seeking induced by cocaine priming increased compared with shams. These results suggest that neonatal ventral hippocampal lesions facilitate instrumental learning for both natural and drug rewards, and reduce inhibitory control over cocaine taking while promoting cocaine seeking and relapse after withdrawal. The findings are discussed in terms of possible developmental or direct effects of the lesions, and both positive reinforcement (substance use vulnerability as a primary disease symptom) and negative reinforcement (selfmedication) theories of substance use comorbidity in schizophrenia.

[Neuropsychopharmacology 27:889-905, 2002] (C) 2002 American College of Neuropsychopharmacology. Published by Elsevier Science Inc.
KEY WORDS: Schizophrenia, Addiction, Dual diagnosis, Dopamine, Nucleus accumbens, Hippocampus

From the Divisions of Molecular Psychiatry and Substance Abuse, West Haven Veterans Administration Hospital and Connecticut Mental Health Center, Department of Psychiatry, Yale University School of Medicine (RAC), and The Seay Center for Basic and Applied Research in Psychiatric Illness Department of Psychiatry, University of Texas Southwestern Medical Center (DWS).

Address correspondence to: R. Andrew Chambers, M.D., Abraham Ribicoff Research Facilities, 3rd Floor, Connecticut Mental Health Center, 34 Park Street, New Haven, CT 06508. Tel.: (203) 9747677; Fax: (203) 974-7897; E-mail: robert.chambers@yale.edu

Received October 31, 2001; revised April 18, 2002; accepted April 30, 2002.

Online publication: 5/2/02 at www.acnp.org/citations/Npp 050202300 .
The high prevalence of substance abuse in the mentally ill poses a major challenge for psychiatric clinicians and mental health care delivery systems. Comorbid substance abuse is related to increased diagnostic uncertainty, symptom severity, medication requirements, risk of violent behavior, and the need for hospital services (Buckley 1998; Dixon 1999). Dual diagnosis in schizophrenia is associated with a particularly high prevalence of amphetamine, cocaine, alcohol, cannabis, and nicotine use (Buckley 1998; DeQuardo et al. 1994; Dixon 1999).

While these drugs have diverse neurochemical and psychoactive properties, they share a common capacity to increase dopamine (DA) levels in the nucleus accum- 
bens (NAc) from dopamine neurons originating in the ventral tegmental area, an event which underlies the positive reinforcing effects of drugs, and can elicit drug-seeking during and/or after withdrawal (Koob and Le Moal 2001; Self 1998; Wise 1998). Other neurotransmitter systems are implicated in the pathophysiology of addiction (Robbins et al. 1998), but DA systems clearly play a major role in certain motivational and neuroadaptive changes associated with chronic exposure to addictive drugs (Gurden et al. 1999; Nestler and Aghajanian 1997; Robinson and Kolb 1999). One of the most enduring neurochemical theories of schizophrenia and psychosis, devised independently from theories of addiction, is also based upon evidence of hyperactivity in the mesolimbic DA system (Crow et al. 1977; Lieberman et al. 1990). Schizophrenia is often marked by a variety of motivational disturbances (Luchins 1992; Skopec et al. 1976) that could involve dysregulation in DA systems that regulate motivated behavior. Indeed, extensive overlap in the neural circuitry putatively involved in both schizophrenia and drug addiction has led to the hypothesis that a common neuropathology underlies substance abuse vulnerability and schizophrenic symptoms (Chambers et al. 2001).

This hypothesis is based on the idea that neuropathological changes in schizophrenia create a predilection for addiction by creating a state of hyper-responsiveness of motivational and DA-related reward circuits, coupled with reduced inhibitory control emanating from cortical structures (Chambers et al. 2001). Neuropathological changes in schizophrenia are most commonly reported in the prefrontal cortex and temporal-limbic regions, particularly in the anterior hippocampal formation, and are thought to produce developmental deficits in local microcircuits that result in symptom manifestation after final maturation in adolescence (Benes and Beretta 2001; Heckers et al. 1998; Weinberger 1999; Weinberger et al. 1994). The anterior ventral hippocampal formation projects to limbic regions such as the prefrontal cortex and the nucleus accumbens, areas that also receive dense dopaminergic innervation from the ventral tegmental area (Groenewegen et al. 1997; Kelley and Domesick 1982; Sesack and Pickel 1990; Verwer et al. 1997). Hippocampal input to the nucleus accumbens exerts a hierarchical gating influence over other excitatory inputs originating in the prefrontal cortex and amygdala (Jay et al. 1995; O'Donnell et al. 1999; O'Donnell and Grace 1995), and also influences activity in ventral tegmental DA neurons (Floresco et al. 2001). Integration of these excitatory and dopaminergic inputs to the nucleus accumbens has been implicated in stimulus-response associations involved in motivational learning and regulation of addictive behavior (Gurden et al. 1999; Mulder et al. 1998; Robbins and Everitt 1996; Self 1998; White and Kalivas 1998). Thus, neuropathological pro- cesses in hippocampal function could profoundly alter temporal-spatial integration of glutamatergic and dopaminergic inputs resulting in both classic schizophrenia symptoms (Schmajuk et al. 2001) and motivational disturbances conducive to substance abuse vulnerability (Chambers et al. 2001).

The neonatal ventral hippocampal lesion (NVHL) model of schizophrenia developed by Lipska and Weinberger produces multiple behavioral disturbances in rats consistent with psychopathology in schizophrenia. NVHL rats show post-pubertal emergence of a behavioral syndrome marked by locomotive hypersensitivity to novelty, stress and pharmacological stimulation of dopamine systems (Lipska et al. 1993). These effects are attenuated by treatment with both typical and atypical antipsychotics (Lipska and Weinberger 1994). NVHL rats tested in adulthood also show sensory gating, cognitive, and working memory deficits (Chambers et al. 1996; Grecksch et al. 1999; Lipska et al. 1995c), and abnormal social interactions (Becker and Grecksch 2000; Becker et al. 1999; Sams-Dodd et al. 1997). Similar neonatal hippocampal lesions produce biochemical and cellular adaptations in brain areas receiving ventral hippocampal input, including the prefrontal cortex and nucleus accumbens, suggesting a developmental impact upon distributed limbic neural networks (Bernstein et al. 1999; Lillrank et al. 1996, 1999; Lipska et al. 1995a; Schroeder et al. 1999).

In this study, the NVHL model was used to investigate motivational responses associated with natural and drug rewards as a possible model of dual diagnosis in schizophrenia. We compared adult NVHL rats with sham-lesioned controls in a series of experiments designed to assess acquisition/extinction of sucrose and cocaine self-administration, drug intake during maintenance of cocaine self-administration, and reinstatement of cocaine seeking to determine potential vulnerability to relapse.

\section{METHODS}

\section{Subjects}

Pregnant Sprague-Dawley rats (Charles River, Kingston, NY) arrived in the laboratory at 14 to 18 days gestation, and were housed in individual cages on a 12hour/day light cycle (lights on 7 A.M.). After delivery, mothers and pups were left undisturbed until postnatal day 7 (PND 7), when male pups weighing 16 to 18 grams were removed for $2-4 \mathrm{~h}$ during neonatal lesioning procedures. After lesioning, pups were returned to their mothers in litters of four to six pups, balanced by lesion status. In some cases, litters were consolidated such that approximately equal numbers of sham and lesioned animals were adopted. Pups were left undisturbed with their mothers under standard feeding/ 
lighting conditions until weaning at PND 23. After weaning, mothers were removed and juvenile pups were maintained in groups of four to six animals balanced with respect to lesion status. All rats were handled prior to behavioral testing. Animals were individually housed at PND 49 one week prior to behavioral testing. All procedures were conducted in accordance with the National Institutes of Health Guide for the Care and Use of Laboratory Animals.

\section{Neonatal Ventral Hippocampal Lesions}

Male pups were randomly assigned to receive either bilateral sham operations or excitotoxic lesions of the ventral hippocampi, according to the protocol developed by Lipska et al. (1993). Pups were anesthetized by hypothermia on ice for 15 to $20 \mathrm{~min}$, and secured with tape to a stereotaxic platform. Head position was maintained by the tape rather than ear bars, such that the dorsal surface was approximately horizontal. A longitudinal incision was made on the dorsal surface of the head, and a Hamilton syringe needle (26 gauge) was lowered through the thin level skull into the ventral hippocampal formation (AP $-3.0 \mathrm{~mm}, \mathrm{ML} \pm 3.5 \mathrm{~mm}$, and VD $\pm 5.0 \mathrm{~mm}$ relative to bregma). Rats assigned to the lesioned group received $3.0 \mu \mathrm{g}$ of ibotenic acid (Sigma, St Louis, MO) in $0.3 \mu$ l of artificial cerebrospinal fluid (CSF) vehicle over $135 \mathrm{~s}$, whereas sham-lesioned rats received only artificial CSF. The needle was left in place for three additional minutes after infusion to prevent backflow through the needle track. The surgical wound was closed using Nexaband veterinary cement and the pups were monitored and warmed with a heating pad before returning to their home cages.

\section{Intravenous Catheter Preparation}

After completion of acquisition/extinction experiments for sucrose reinforcement, adult rats underwent surgical placement of a chronic indwelling jugular catheter composed of Silastic tubing (Green Rubber, Woburn, MA) for cocaine self-administration as described previously (Sutton et al. 2000). The jugular catheter passed subcutaneously to exit the back through 22gauge cannulae (Plastics One, Roanoke, VA), embedded in cranioplastic cement and secured with Marlex surgical mesh (Bard Inc., Cranston, RI). Animals received a prophylactic injection of penicillin $(60,000 \mathrm{IU}$, i.m.), and were allowed one week to recover before cocaine self-administration testing. Catheters were flushed daily with $0.2 \mathrm{ml}$ heparinized ( $20 \mathrm{IU} / \mathrm{ml}$ ) bacteriostatic saline containing gentamycin sulfate $(0.33 \mathrm{mg} /$ $\mathrm{ml}$ ), and checked once a week for catheter blockage with $1 \mathrm{mg} / 0.1 \mathrm{ml}$ methohexital sodium i.v. (Henry Schein, Indianapolis IN) which produces a brief period of anesthesia.

\section{Apparatus}

All experimental tests were conducted in operant test chambers (Med Associates, Inc., St. Albans, VT), equipped with two levers located $3 \mathrm{~cm}$ above the test chamber floor, cue lights located just above each lever, and a house light located opposite the wall containing the levers. Each chamber was enclosed in a sound-attenuating cubicle equipped with an infusion pump assembly consisting of a Razel Model A pump (Med Associates, Inc.) and a 10-ml glass syringe. The syringe was connected to a fluid swivel (Stoelting, Wooddale, IL) by Teflon tubing. Tygon tubing connected the swivel to the animal's catheter assembly and was secured by a metal spring connector. A cue light was illuminated continuously above the active lever during acquisition of sucrose reinforcement, and sucrose pellets were delivered in a food trough located between the active and inactive levers. During cocaine self-administration, cocaine injections were delivered over $4 \mathrm{~s}$ in a $0.08-\mathrm{ml}$ volume of sterile-filtered saline, concurrent with illumination of the active lever cue light; responses during the injection were recorded but produced no programmed consequence. Each injection was followed by an additional 10-s time-out period signaled by lights out, so that the injection/time-out period totaled $14 \mathrm{~s}$. Injection dose was adjusted by changing the stock concentration from 1.25 to $5 \mathrm{mg} / \mathrm{kg} / \mathrm{ml}$.

\section{Behavioral Procedures}

Each rat was tested in a series of seven experiments all conducted five days per week in the same test chamber and at the same approximate time during the light cycle, beginning on PND 56 and ending approximately 80-100 days later (Figure 1). Left or right levers were randomly assigned as active for each rat during sucrose reinforcement, and the alternate lever was used for cocaine reinforcement. Animals were weighed daily, and levers were cleaned with ethanol after each test session.

Habituation of Spontaneous Lever Pressing. Rats were maintained at $90 \%$ of their initial body weight during habituation of spontaneous lever pressing and acquisition of sucrose self-administration. Initial spontaneous (non-reinforced) lever press responding was recorded at both levers in a 1-h test session. This procedure continued in daily 1-h tests until responding habituated to a criterion of 10 or less total lever presses (at both levers) in each rat. The latency (number of sessions) to reach habituation criterion was recorded; animals that required more than 10 sessions to meet habituation criterion received a maximum latency score of 10 sessions to reduce outlier variance.

Acquisition of Sucrose Pellet Self-administration. After habituation of spontaneous lever press behavior to criterion, animals were allowed to acquire sucrose self- 


\section{Experimental Schedule}
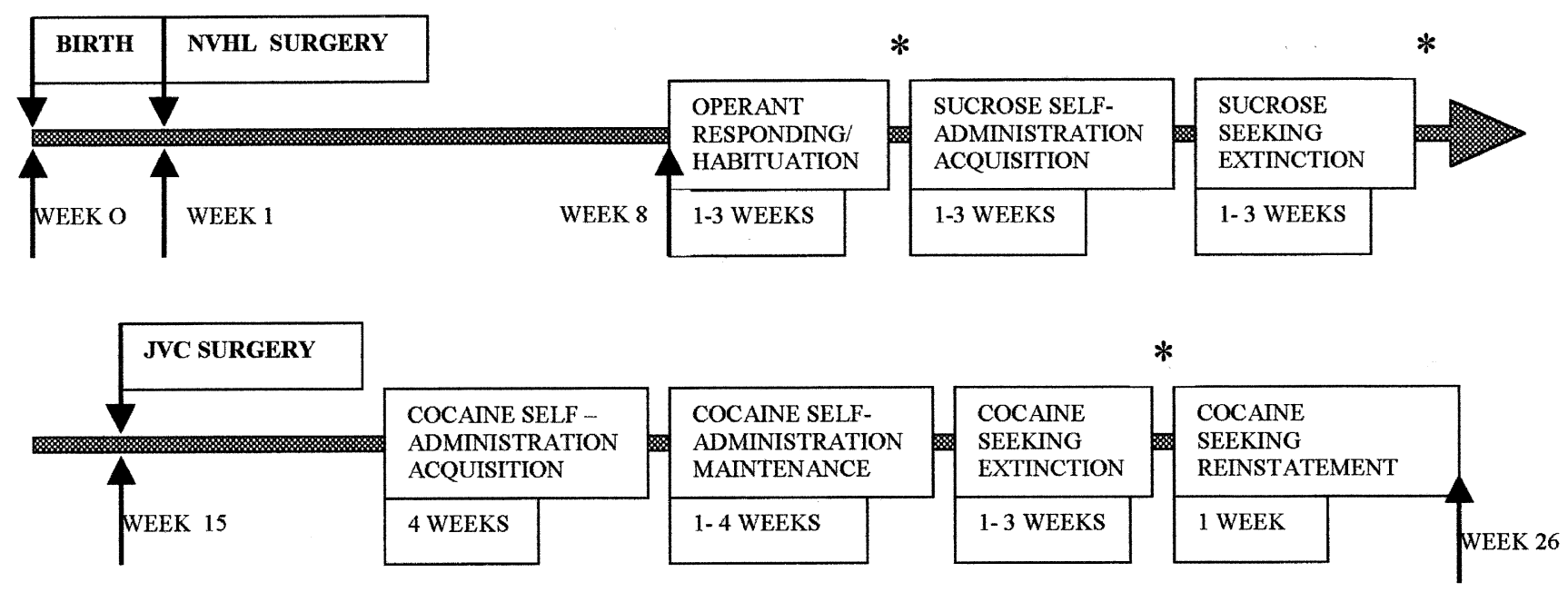

Figure 1. Experimental time line. Vertical arrows indicate age of rat in weeks when surgeries and experimentation were conducted. Approximate duration of each of seven experimental phases is listed below the line. Asterisks indicate times when lever-press response rates were either habituated or extinguished to similar baseline levels prior to further testing.

administration (45 mg sucrose pellets, Bioserve) on a fixed ratio 3 (FR3) schedule of reinforcement with the active lever cue light on continuously and the house lights off. Test sessions lasted for $2 \mathrm{~h}$, or until rats had received 100 sucrose pellets (300 active lever presses). Sessions were repeated until individual rats met acquisition criterion, defined as the third of three consecutive sessions in which the maximum of 100 sucrose pellets was delivered. The latency (number of sessions) to reach acquisition criterion was recorded. When rats failed to press the lever at least once during the previous test session, a single sucrose pellet was placed on the active lever at the onset of the next test session. Rats were disqualified from the rest of the study if acquisition criterion was not reached after 15 consecutive test sessions. The latency to consume 100 pellets was measured on the last two of three consecutive days on which all 100 pellets were earned, and average latencies between groups were compared.

Extinction of Sucrose-seeking Responses. Rats were fed ad lib during extinction testing and the remainder of the experiment. Extinction testing was conducted for $2 \mathrm{~h} /$ session in the absence of sucrose reinforcement, but with house lights off and a cue light on similar to the acquisition phase. Extinction test sessions were repeated until rats had reached extinction criterion defined as $\leqslant 15$ responses/session at the sucrose-paired lever. The number of responses at the sucrose-paired lever during the first and second test sessions, and the number of sessions to reach extinction criterion were recorded. A minimum of two and a maximum of 15 extinction test sessions were required to achieve this criterion for all rats. After extinction testing, rats were surgically prepared with chronic indwelling intravenous catheters as described above.

Acquisition of Cocaine Self-administration. Following extinction of responding on the sucrose-paired lever, and one week after surgical catheter preparation, rats were tested for acquisition of cocaine self-administration by responding at the alternate lever used in sucrose experiments on a FR 1 reinforcement schedule. Acquisition testing was conducted in five daily $2 \mathrm{~h}$ sessions/ week over a 4-week period, with ascending doses available each week for three weeks $(0.1,0.2,0.4 \mathrm{mg} / \mathrm{kg} /$ injection), followed by a second week at the $0.4 \mathrm{mg} / \mathrm{kg} /$ injection dose. Acquisition criterion was defined as selfadministering 30 or more cocaine injections for three consecutive sessions. A maximum of 120 cocaine injections was available in each test session and sessions were terminated upon reaching this maximum. This ceiling was implemented to preclude large betweengroup differences in total cocaine intake that could alter subsequent behavioral measures as an indirect rather than direct consequence of the lesions. For each session, the total number of active lever responses was recorded, as were the corresponding number of active le- 
ver presses that produced cocaine injections, and the number that occurred after the onset of each injection during the 14-s time period. These latter measures reflect the amount of total active lever responses that result in cocaine intake and the level of response perseveration during the cocaine injections, respectively. No priming injections were given during acquisition, but a single food pellet was placed on the active lever at the session onset for rats that failed to respond on the active lever in the previous session.

Maintenance of Cocaine Self-administration. Rats were allowed to continue cocaine self-administration (0.4 $\mathrm{mg} / \mathrm{kg} /$ injection) in daily 2-h test sessions until they had self-administered at least 25 injections/session for a total of 15 sessions. Rats that failed to achieve criterion for acquisition during the initial 4-week acquisition phase (four sham and two lesioned) were food restricted to $90 \%$ current body weight and trained to selfadminister cocaine with priming injections until they achieved 15 sessions of self-administration as described above, with at least five final sessions under ad lib conditions. The last five days of cocaine self-administration extinction/reinstatement testing served as a baseline.

Extinction of Cocaine-seeking Responses. Extinction test sessions commenced three days after the last self-administration test in daily $2-\mathrm{h} /$ day sessions with both levers inactive, (no cues or injections delivered; house light on). Rats were tested for a minimum of two extinction sessions, or until extinction criteria of $\leqslant 15$ drug-paired lever, and $\leqslant 30$ total lever responses in a single 4 -h session were achieved. Initial extinction responding in the first two sessions, and the latency to achieve extinction criteria were analyzed and shown graphically. Animals requiring $>15$ sessions to meet extinction criteria received a maximum latency score of 15 sessions to reduce outlier variance, but training continued until criteria was met.

Reinstatement of Cocaine-seeking Responses. Reinstatement test sessions were conducted three to 10 days after reaching extinction criteria such that all animals were tested approximately two weeks since their last cocaine self-administration test session. Each reinstatement test was conducted on a separate day for a 1-h period following an additional $2 \mathrm{~h}$ of extinction conditions. Reinstatement testing commenced following an ip priming injection of saline or cocaine ( 5 and $15 \mathrm{mg} / \mathrm{kg}$ ) given in counterbalanced order across sessions. Rats showing high levels of lever responding ( $>30$ sum of both levers) in the 1-h baseline period preceding the injections were not tested. Non-reinforced lever-press responses were recorded at both drug-paired and inactive levers during the final hour of reinstatement testing.

\section{Lesion and Data Analysis}

After completing behavioral experiments, animals were anesthetized by chloral hydrate, decapitated, and brains rapidly frozen. Twenty $\mu \mathrm{m}$ horizontal brain sections spaced approximately 400 micrometers apart were collected on a cryostat, mounted, and fixed with chloroform followed by Nissl staining with $0.05 \%$ thionine. The extent of the lesions was determined by microscopic assessment of thionine-stained sections from -3.30 to $-5.80 \mathrm{~mm}$ posterior to bregma. Animals with lesions extending beyond the hippocampus to adjacent regions, or with only unilateral damage to the hippocampus, were excluded from the statistical analysis, but are reported in Table $1(n=5)$.

Unitary behavioral measures such as latency to habituate, acquire, or extinguish responding, and total cocaine consumed, compared sham and lesioned animals with 2 -tailed $t$-tests. For acquisition of cocaine self-administration, each week of testing at a given injection dose was first analyzed by 3-way ANOVA, with repeated measures on test day and lever (active and inactive). Significant effects of Lever or Lesion group X Lever interactions were followed up by analysis of each lever independently by 2-way ANOVA (Group X Test day). Similarly, given that total active lever responding includes both cocaine injections and time-out active lever responses, average daily cocaine injections/week and time out responses/week, and total cocaine intake/week were each analyzed by 2-way ANOVA with repeated measures on test week. A similar 3-way followed by 2-way ANOVA protocol was used to analyze baseline responding following acquisition of cocaine self-administration, extinction from sucrose and cocaine reinforcement, and cocaine-induced reinstatement. Cocaine acquisition criteria, incidence (percentage) of test sessions when the maximal available injections were taken, and the incidence of perseverative $(>100)$ inactive lever responses during cocaine self-administration were compared by non-parametric Mann-Whitney $U$ tests. Data are presented as mean \pm SEM. Statistical differences of $p<.05$ are reported as significant. During the course of the experiment, one bilateral NVHL rat died from cocaine overdose and was included in the analyses of sucrose, but not cocaine, self-administration experiments. One of the 13 sham-lesioned rats failed to acquire cocaine self-administration during the acquisition or maintenance phases, and was excluded from analyses beyond acquisition of cocaine self-administration. Two of $10 \mathrm{NVHL}$ and one of 12 shams were excluded from the reinstatement analysis due to high $(>30)$ baseline responding in the $1 \mathrm{~h}$ period preceding reinstatement injections. One unilateral-lesioned rat died in surgery, and another lost catheter patency during acquisition of cocaine self-administration; cocaine self-administration data from the three remaining unilateral lesioned rats are shown in Table 1. 
Table 1. Results in non-reinforced responding, sucrose and cocaine self-administration (mean \pm SEM).

Spontaneous non-reinforced responding

$$
\text { Total initial lever }
$$$$
\text { presses } / 1 \mathrm{~h}
$$

$$
\text { Sham }(\mathrm{N}=13) \quad 41.0 \pm 7.7
$$

Lesioned $(\mathrm{N}=11) \quad 68.7 \pm 22.1$

Unilateral $(\mathrm{N}=5) \quad 34.8 \pm 11.1$

Acquisition of sucrose reinforcement

\begin{tabular}{lr} 
& $\frac{\text { Acquisition latency }}{\text { (\# sessions) }}$ \\
\cline { 2 - 2 } Sham $(\mathrm{N}=13)$ & $6.54 \pm 0.78$ \\
Lesioned $(\mathrm{N}=11)$ & $4.09 \pm 0.28$ \\
Unilateral $(\mathrm{N}=5)$ & $6.8 \pm 2.11$
\end{tabular}

\begin{tabular}{|c|c|c|c|}
\hline \multirow[b]{3}{*}{ Sham $(\mathrm{N}=13)$} & \multicolumn{2}{|c|}{ Initial extinction responding (First session) } & \multirow{2}{*}{$\begin{array}{l}\text { Extinction latency } \\
\text { (\# sessions) }\end{array}$} \\
\hline & $\underline{\text { Sucrose-paired lever }}$ & Inactive lever & \\
\hline & $200.5 \pm 21.4$ & $\overline{45.9 \pm 21.7}$ & $5.46 \pm 0.98$ \\
\hline Lesioned $(\mathrm{N}=11)$ & $174.8 \pm 34.5$ & $44.9 \pm 13.7$ & $5.45 \pm 1.27$ \\
\hline Unilateral $(\mathrm{N}=4)$ & $225.8 \pm 59.8$ & $33.3 \pm 14.0$ & $10.25 \pm 2.75$ \\
\hline
\end{tabular}

Extinction from sucrose reinforcement

\author{
Habituation latency (\# sessions) \\ $2.38 \pm 0.30$ \\ $4.18 \pm 1.02$ \\ $2.60 \pm 1.12$
}

Acquisition of cocaine self-administration

Drug-paired lever responses

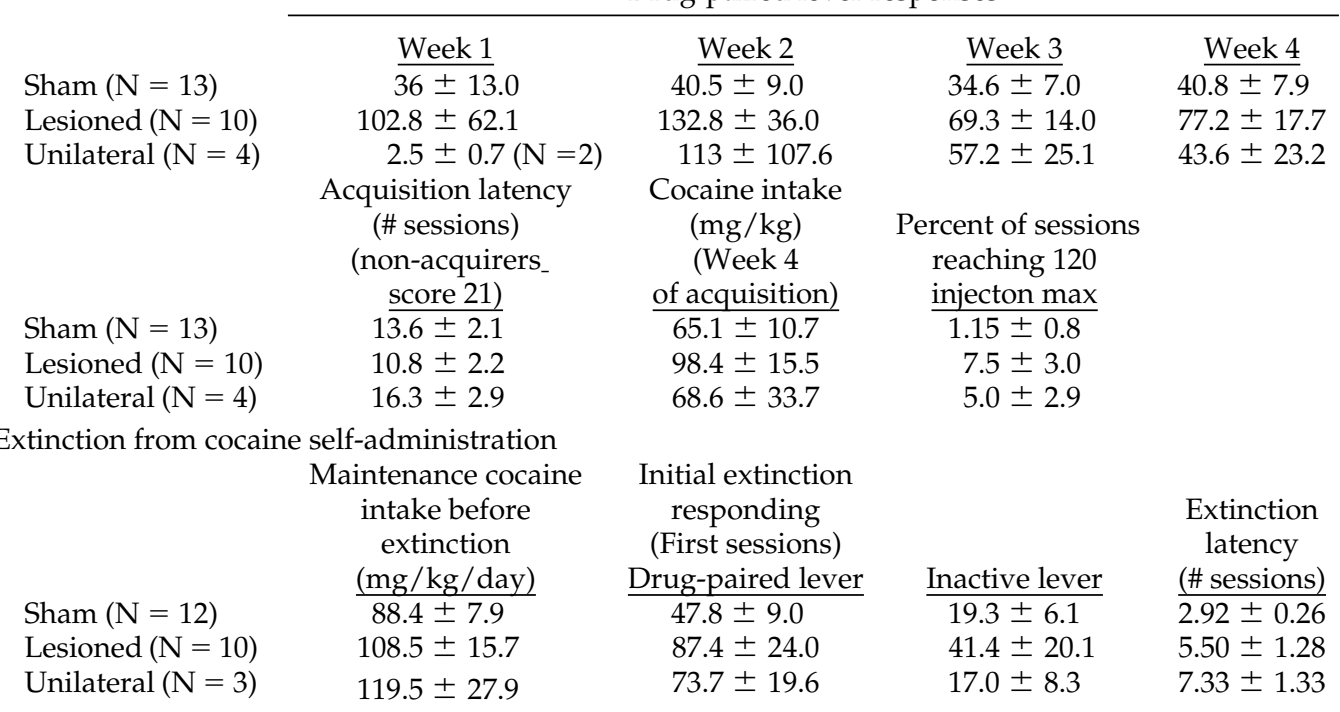

\begin{tabular}{|c|c|c|c|c|c|c|}
\hline & \multicolumn{6}{|c|}{ Drug-paired lever responses / $1 \mathrm{~h}$} \\
\hline & Baseline & Saline & Baseline & Coc $5 \mathrm{mg} / \mathrm{kg}$ & Baseline & Coc $15 \mathrm{mg} / \mathrm{kg}$ \\
\hline Sham $(\mathrm{N}=11)$ & $\overline{2.5 \pm 0.8}$ & $3 . \overline{9 \pm 1.5}$ & $\overline{2.1 \pm 1.1}$ & $16.9 \pm 3.6$ & $\overline{2.3 \pm 0.8}$ & $44.0 \pm 10.9$ \\
\hline Lesioned $(\mathrm{N}=8)$ & $2.6 \pm 1.6$ & $2.8 \pm 1.0$ & $1.9 \pm 0.6$ & $51.9 \pm 11.4$ & $2.6 \pm 1.7$ & $107.6 \pm 51.0$ \\
\hline Unilateral $(\mathrm{N}=3)$ & (not listed due to & & & & & \\
\hline
\end{tabular}

Cocaine-induced reinstatement

\section{RESULTS}

\section{Neonatal Ventral Hippocampal Lesions}

Sixteen neonatal rat pups received ibotenic acid lesions while 13 pups received sham surgery. Figure 2 shows the extent of the lesions in eleven of the ibotenic acidlesioned rats that showed bilateral damage confined to hippocampal regions. Positive hippocampal damage was scored upon visualization of neuronal loss (gliosis) in hippocampal tissue in at least two consecutive sections spaced 400 micrometers apart. The smallest lesion was confined to the posterior pole of dorsal and ventral
CA3 regions, whereas the largest extended into the ventral CA1 and subiculum, and dorsal anterior CA3. The lateral ventricles were usually enlarged. Slight gliosis was present on the lateral aspect of thalamic capsule adjacent to the hippocampus in five cases. These animals were used in the behavioral analysis. Five of the 16 NVHL rats showed only unilateral damage to the hippocampus; behavioral measures for these animals are shown in Table 1 . The 13 sham-lesioned rats showed no evidence of hippocampal tissue damage. 

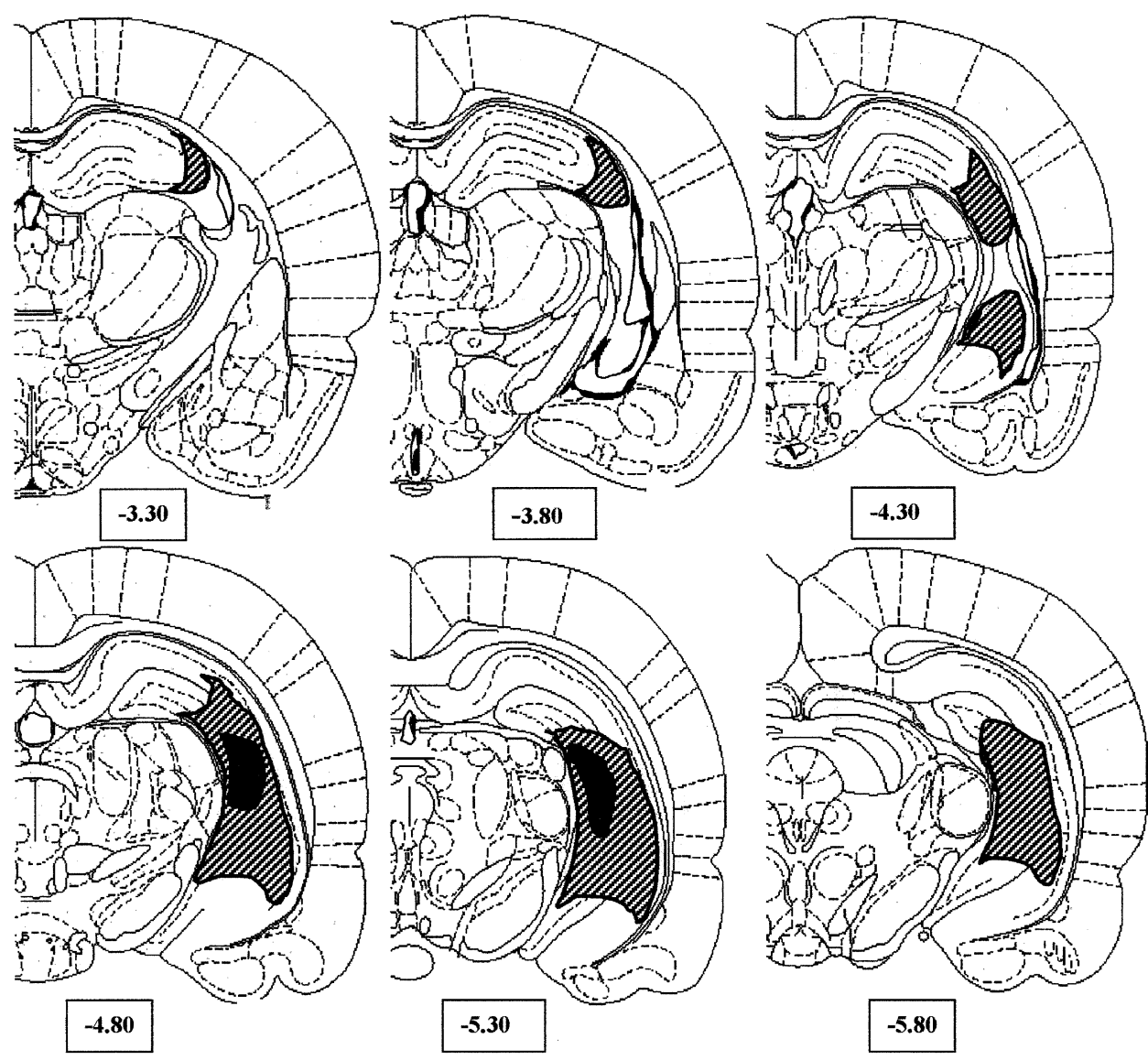

Figure 2. Hemi-coronal representations showing the extent of neonatal ibotenic acid-induced lesions. Striped areas represent largest extent of the lesion (on either side) in the bilaterally lesioned group, while the black areas represent the smallest lesion area. The majority of lesioned rats had lesion sizes closer to the large extreme. Numbers below the sections indicate distance of sections posterior to bregma based on stereotaxic coordinates of Paxinos and Watson.

\section{Habituation of Spontaneous Lever Pressing}

NVHL and sham rats attained similar weights by PND 56 prior to the onset of behavioral testing (NVHL $390.4 \pm$ 6.6; sham, 394.9g $\pm 13.0 \mathrm{~g}$ ). Spontaneous non-reinforced lever-press responses at both levers during the initial exposure to the test chambers averaged $68.7 \pm 22.1$ for NVHL and $41.0 \pm 7.7$ for sham rats, but did not significantly differ $\left(t_{22}=1.26, p>.05\right)$ under restricted diet conditions used for subsequent sucrose self-administration (Figure 3, panel A). NVHL rats required an average 1.8 more sessions to reach the habituation criteria than shams (Figure 3, panel B), producing marginally different habituation latency scores $\left(t_{22}=1.81, p=.08\right)$. Two lesioned rats required 11 and 18 sessions to reach criteria for habituation, but were scored as 10 according to predetermined protocol (see methods).

\section{Acquisition/Extinction of Sucrose Self-Administration}

Following habituation of spontaneous lever pressing to $\leqslant 10$ responses/hour in all NVHL and sham animals, NVHL animals acquired sucrose self-administration more readily than shams $\left(\mathrm{t}_{22}=-2.75, p<.05\right)$, requiring an average of 2.45 fewer training sessions to reach acquisition criterion (Figure 3, panel C). Over the last two acquisition sessions, there were no significant differences in the time required to consume 100 sucrose pellets, although sham rats tended to require less time, $16.2 \pm 1.8 \mathrm{~min}$, compared with $24.2 \pm 4.4 \mathrm{~min}$ for NVHLs.

Responding during extinction from sucrose reinforcement failed to differ between NVHL and shams $\left(\mathrm{F}_{1,22}=0.09, p>.05\right)$, although a main effect of lever $\left(\mathrm{F}_{1,22}=50.45, p<.001\right)$ and test session $\left(\mathrm{F}_{1,22}=24.48\right.$, $p<.001)$ was found, reflecting greater responding at the active lever on the first test session among both groups (Figure 3, panel D). Thus, extinction responding at both the sucrose-paired and inactive levers was similar in NVHL and shams in the initial two extinction tests. Moreover, the number of sessions required to achieve extinction criteria failed to differ between NVHL and shams (Figure 3, panel E; $\mathrm{t}_{22}=-0.004, p>.05$ ). Therefore, NVH lesions facilitated acquisition of sucrose reinforcement, but fail to alter the magnitude or perseverance of responding during extinction.

\section{Acquisition of Cocaine Self-administration}

Following extinction of sucrose self-administration to $\leqslant$ 15 responses/test session, rats were prepared with in- 


\section{Spontaneous Non-Reinforced Responding \\ SHAM $(\mathrm{N}=13)$, NVHL $(\mathrm{N}=11)$}

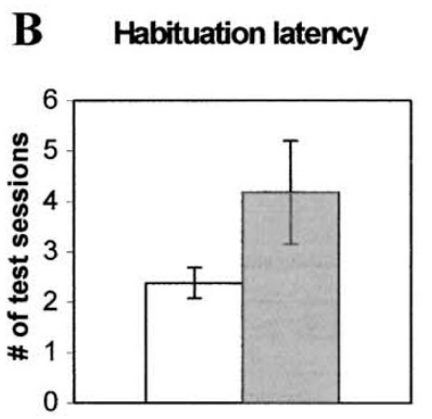

\section{Acquisition/Extinction of Sucrose Reinforcement $\operatorname{SHAM}(\mathrm{N}=13)$, NVHL $(\mathrm{N}=11)$}

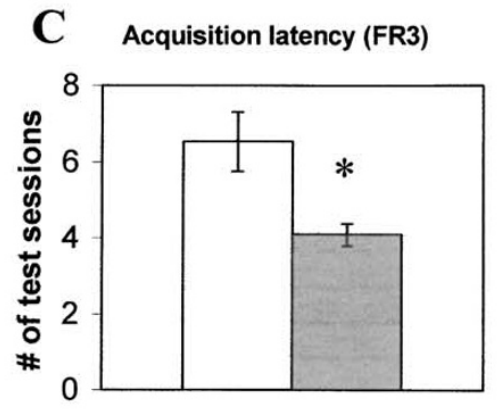

D

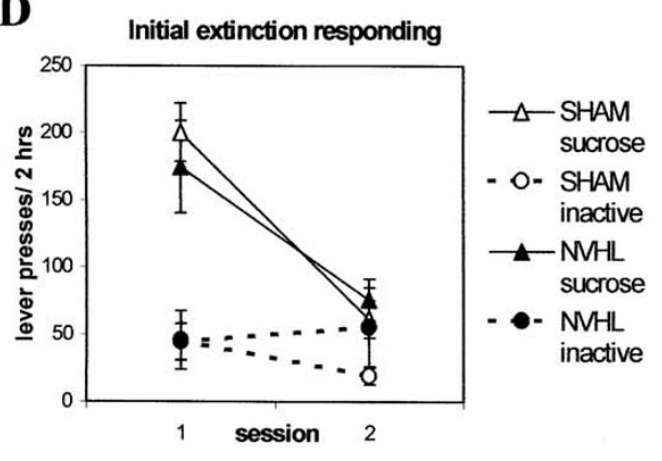

E

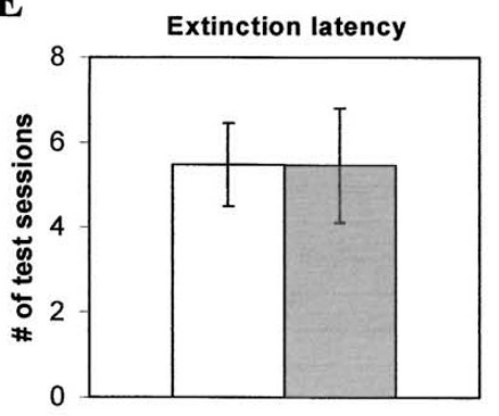

Figure 3. Profile of spontaneous and sucrose-reinforced responding in NVHL and sham rats. NVHL rats show non-significant trends for (A) increased spontaneous leverpress responses in an initial 1-h test, and (B) latency to reach habituation criterion (number of sessions to $\leqslant 10$ total lever responses/hour). C. NVHL animals acquire sucrose pellet self-administration (FR3) with shorter latencies to criterion (three consecutive sessions receiving the maximum 100 sucrose pellets) relative to shams. D. Response rates during the initial two extinction tests are similar in NVHL and sham rats at both sucrose-paired and inactive levers. E. The latency to reach extinction criterion is also similar (number of sessions to reach $\leqslant 15$ responses on sucrose-paired lever). Data are presented as the mean \pm SEM, ${ }^{*} p<.05$ by 2 -tailed $t$-test. travenous catheters and allowed one week to recover, and then allowed to acquire cocaine self-administration by pressing the lever that was inactive during previous sucrose reinforcement tests. Ascending doses were made available each week for three weeks $(0.1,0.2$ and $0.4 \mathrm{mg} / \mathrm{kg} /$ injection), followed by an additional week of testing at the $0.4 \mathrm{mg} / \mathrm{kg} /$ injection dose. A main effect of Lesion and/or Lesion $X$ Lever interaction was found for week 2 (Lesion: $\mathrm{F}_{1,21}=8.94, p<.01$; Lesion $\mathrm{X}$ Lever: $\mathrm{F}_{1,21}=5.94, p<.05$ ), week 3 (Lesion: $\mathrm{F}_{1,21}=3.844$, $p=.063$; Lesion $\mathrm{X}$ Lever: $\left.\mathrm{F}_{1,21}=6.37, p<.05\right)$, and week 4 (Lesion: $\mathrm{F}_{1,21}=7.24, p<.05$; Lesion $\mathrm{X}$ Lever: $\mathrm{F}_{1,21}=$ $1.73, p>.05)$, while neither of these were significant in week 1 (Lesion: $\mathrm{F}_{1,21}=1.14, p>.05$; Lesion $X$ Lever: $\mathrm{F}_{1,21}=$ 1.69, $p>$.05). Subsequent 2-way ANOVA (Lesion $X$ Session) on separate active and inactive levers found that active lever responses were increased in NVHL rats in week $2\left(\mathrm{~F}_{1,21}=7.83, p<.05\right)$, week $3\left(\mathrm{~F}_{1,21}=5.67\right.$, $p<.05)$, and week $4\left(\mathrm{~F}_{1,21}=5.00, p<.05\right)$, as depicted in Figure 4, panel A. Inactive lever responding failed to differ between NVHL and sham rats in any week of acquisition testing ( $p$ values $>0.10$ ). Figure 4, panel B, shows that higher active lever responding in lesioned rats was due primarily to response perseveration during the 14-s injection/time-out periods $\left(\mathrm{F}_{1,21}=6.71, p<\right.$ .05), rather than the number of cocaine injections taken 


\section{Acquisition of Cocaine Self-Administration SHAM $(\mathrm{N}=13)$, NVHL $(\mathrm{N}=10)$}
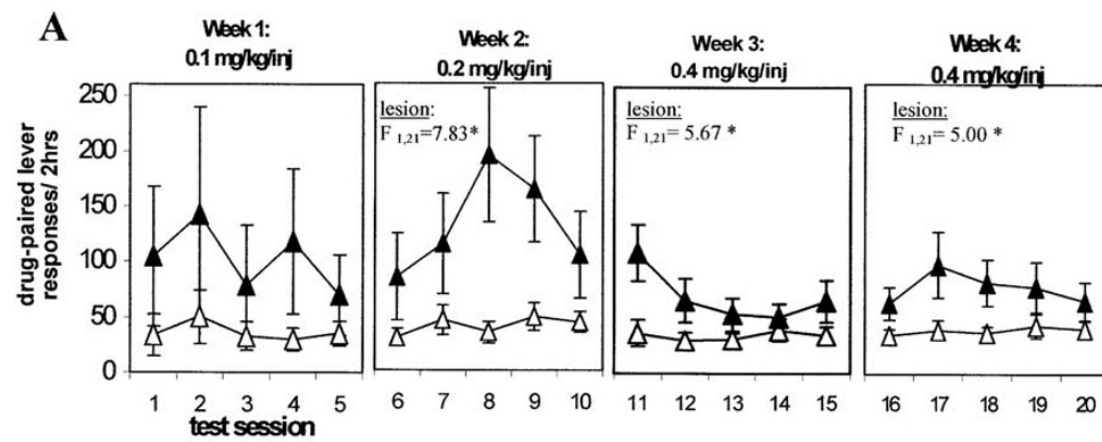

B

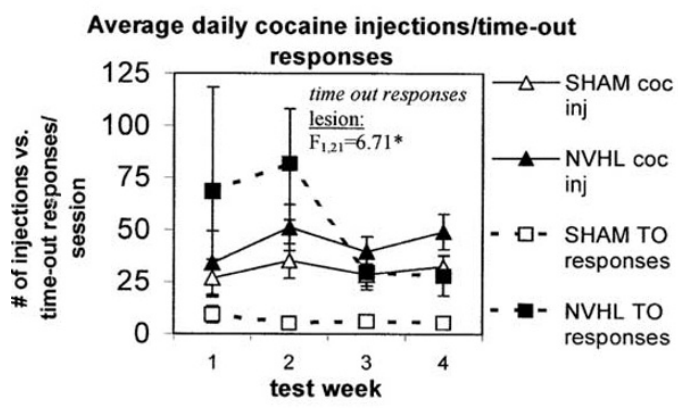

D

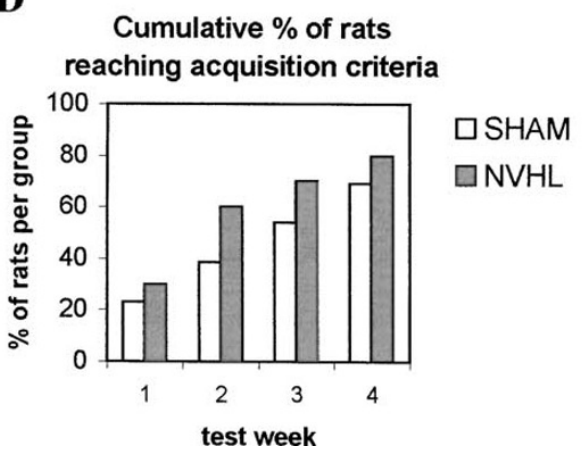

C

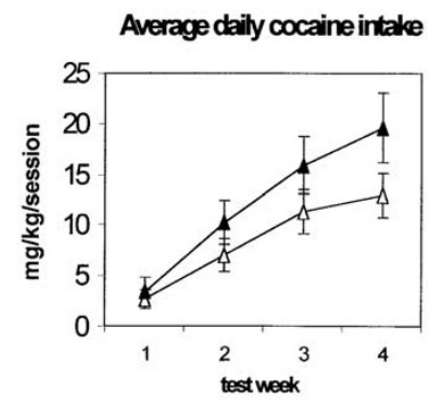

$\mathbf{E}$ Acquisition latency (FR1)

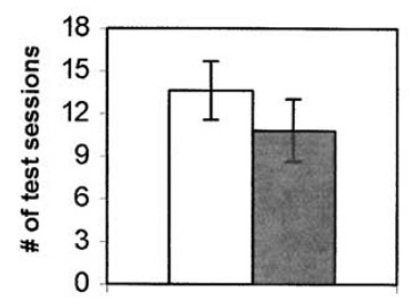

Figure 4. Acquisition of cocaine self-administration (FR1:TO $14 \mathrm{~s}$ ) in NVHL and sham rats over four weeks of ascending dose availability. A. NVHL rats respond more at the active lever beginning the second week at an injection dose of 0.2 $\mathrm{mg} / \mathrm{kg}$, and continue through weeks 3 and $4(0.4 \mathrm{mg} / \mathrm{kg} /$ injection). B. Increases in total active lever responding in NVHL rats shown in (A) are reflected by increases in average daily time-out (TO) responses during the 14-s injection/time out periods, and not by increases in the number of injections taken. C. Average daily cocaine intake $(\mathrm{mg} / \mathrm{kg})$ increased in both groups, with a non-significant trend for early escalation in NVHL rats (but see Figure 6). D. More than $50 \%$ of NVHL rats reach criterion for acquisition of cocaine self-administration $(\geqslant$ 30 cocaine injections/session for three consecutive sessions) by the end of the second test week, while shams require three weeks and a higher injection dose to achieve $50 \%$ acquisition. E. However, the mean latency to acquire cocaine selfadministration was not significantly shorter in NVHL rats. Data are presented as the mean \pm SEM, ${ }^{*} p<.05$ by 2 -way ANOVA with repeated measures on test day or week.
$\left(\mathrm{F}_{1,21}=1.39, p>.05\right)$. However, this non-reinforced component of total active lever responding became less prominent when the training dose was increased during weeks 3 and 4 of acquisition testing, as reflected by the marginally significant interaction between lesion and test week $\left(\mathrm{F}_{3,19}=2.943, p=.059\right)$. In contrast, sham animals engage in markedly less time-out responding throughout training. While average daily cocaine intake tended to increase in lesioned rats during weeks 3 and 4 , this trend did not reach statistical significance (Figure 4 , panel C).

The percent of NVHL rats reaching acquisition criterion was $60 \%$ by the end of the second week of training, whereas only $38 \%$ of sham rats had reached this crite- rion (Figure 4, panel D). By the fourth week of testing, $69 \%$ of shams had reached acquisition criterion compared with $80 \%$ of NVHL rats, reflecting the four of 13 shams and two of 10 lesioned rats that failed to acquire by the end of the 4-week test, respectively. However, there was no significant difference in the percentage of animals that acquired at each week by non-parametric analysis. Similarly, and in contrast to sucrose self-administration, there was no difference in acquisition latencies for cocaine self-administration in NVHL and sham rats that acquired cocaine self-administration (Figure 4, panel E). Thus, the primary effect of NVH lesions was response perseveration during initial acquisition of cocaine self-administration at low injection 
doses. However, NVHL rats also showed a significantly greater incidence of binge-like self-administration, as indicated by a greater percentage of acquisition tests where the maximum allotted 120 drug injections was self-administered (Mann-Whitney $\mathrm{U}, p<.05$ ). This occurred in $7.5 \%$ of all test sessions for NVHL rats, and in $1.1 \%$ sessions for shams (Figure 5, panel A). The limit on the maximum allotted injections/session could account for non-significant divergence in weekly cocaine intake as the total amount of cocaine available/week increased from 60 to $240 \mathrm{mg} / \mathrm{kg}$. There was no effect of lesion status on the incidence of response perseveration at the inactive lever (percentage of tests with $>100$ inactive lever responses) during cocaine self-administration (Figure 5, panel B).

\section{Maintenance of Cocaine Self-administration}

Four sham and two NVHL rats that failed to acquire cocaine self-administration received additional training until they had achieved equivalent cocaine self-administration experience (see Methods); one sham rat could not be trained to self-administer cocaine, and was not tested further. Baseline cocaine intake over the final five days of cocaine self-administration prior to extinction testing is shown in Figure 6, panel A. NVHL and sham rats continued to self-administer similar amounts of cocaine, averaging $21.7 \pm 3.1$ relative to $17.7 \pm 1.6 \mathrm{mg}$ / $\mathrm{kg} /$ day in shams. However, NVHL rats continued to exhibit significantly higher response perseveration during the post-injection time-out periods $\left(\mathrm{F}_{1,20}=7.71, p<\right.$
.05) despite stabilization of self-administration patterns, as depicted graphically in Figure 6, panel A, and in examples of individual response records in Figure 6, panel B. NVHL rats consumed an average grand total of $399.31 \pm 49.9 \mathrm{mg} / \mathrm{kg}$ over the course of acquisition and maintenance phases of cocaine self-administration, whereas shams consumed $329.9 \pm 18.1 \mathrm{mg} / \mathrm{kg}$, although these totals did not differ statistically.

\section{Extinction/Reinstatement of Cocaine Self-administration}

In contrast to extinction from sucrose reinforcement, there was a significant Lesion $X$ Lever interaction $\left(\mathrm{F}_{1,20}=\right.$ $8.38, p<.01)$ in the initial two extinction tests from cocaine self-administration, and the main effect of lesion was marginally significant $\left(\mathrm{F}_{1,20}=3.56, p=.07\right)$. Separate analysis of each lever found significantly greater responses at the cocaine-paired lever in NVHL rats $\left(\mathrm{F}_{1,20}=5.51, p<.05\right)$, but not at the inactive lever (Figure 7, panel A). NVHL rats also averaged 2.6 more test sessions to reach extinction criterion than shams (Figure 7, panel B), resulting in significantly longer extinction latencies $\left(t_{20}=2.15, p<.05\right)$. Following achievement of extinction criteria, and about two weeks after the last cocaine self-administration session, animals were tested for cocaine-induced reinstatement of $\mathrm{CO}^{-}$ caine seeking. Initial 3-way ANOVA revealed that a single intraperitoneal priming injection of cocaine dosedependently increased responding (main effect of dose: $\left.\mathrm{F}_{2,16}=8.48, p<.01\right)$, with a significant effect of Lesion

\section{Group Extremes in Responding During Cocaine Acquisition}
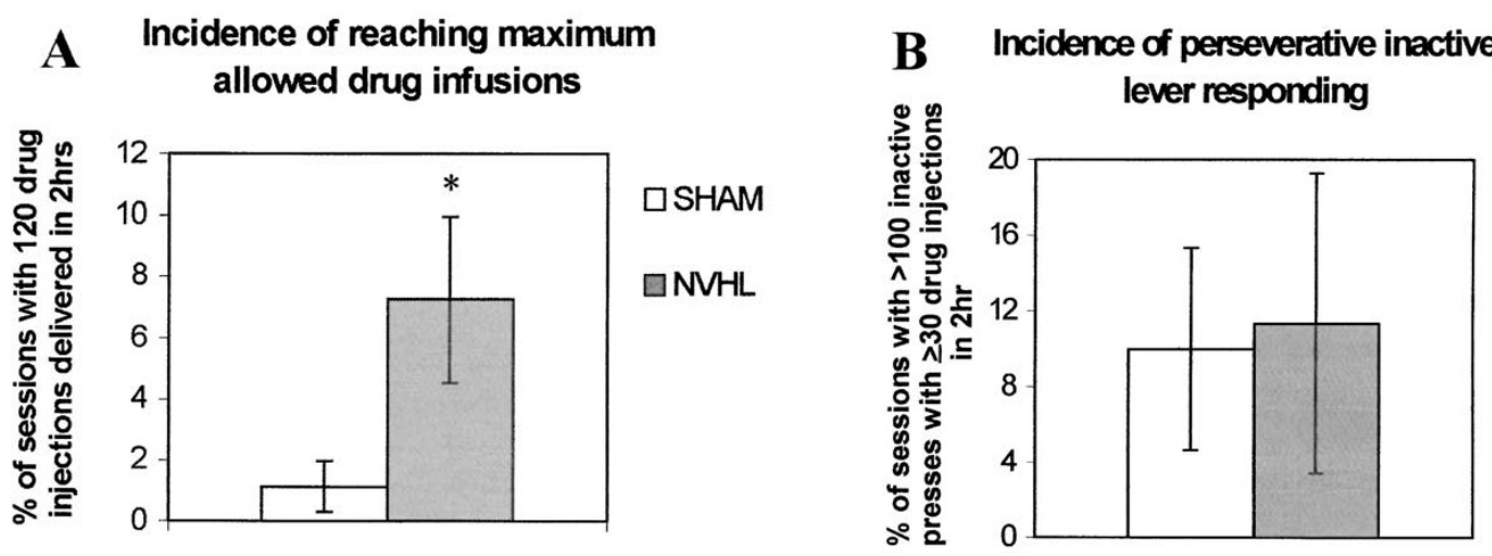

Figure 5. Incidence of binge-like cocaine self-administration and response perseveration at the inactive lever in NVHL and sham rats. A. NVHL rats show a greater incidence of obtaining the maximum number of cocaine injections available (120 per session) than shams over the 4-week acquisition period. Data are expressed as the mean \pm SEM percentage of total tests/animal. B. The incidence of response perseveration at the inactive lever during active cocaine self-administration is similar between NVHL and sham rats ( $\geqslant 100$ responses concomitant with $\geqslant 30$ cocaine injections). ${ }^{*}<.05$ by Mann-Whitney $\mathrm{U}$ test. 


\section{Baseline Cocaine Self-Administration Prior to Extinction SHAM $(\mathrm{N}=12)$, NVHL $(\mathrm{N}=10)$}

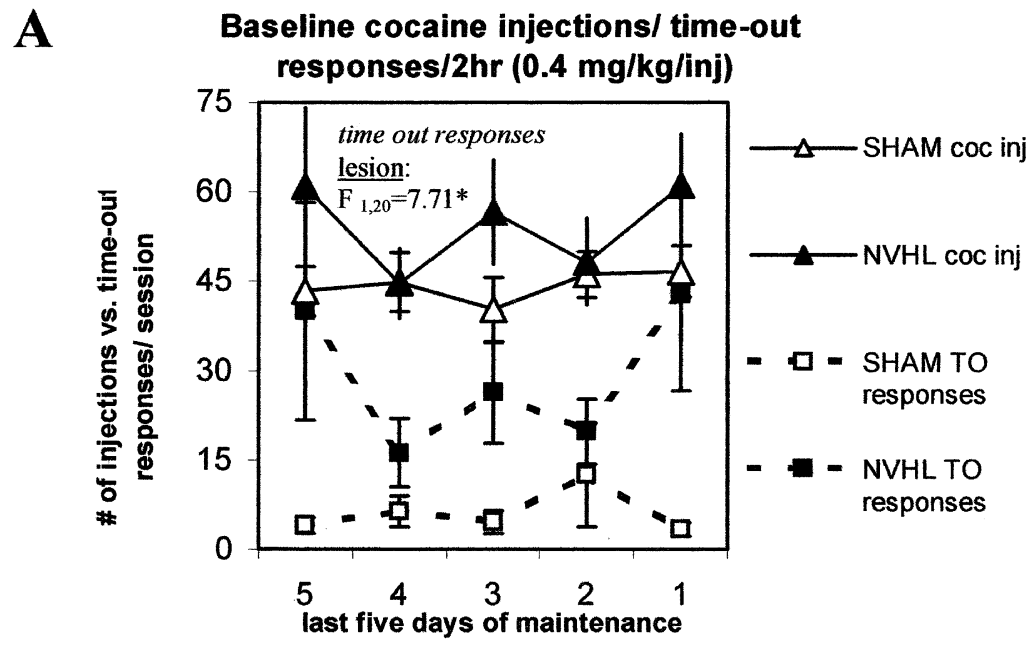

B

\section{Typical response patterns over 30 minutes}

$\begin{array}{ll}\text { NVHL } & \begin{array}{l}\text { Drug } \\ \text { injections }\end{array} \\ \text { rat } & \begin{array}{l}\text { Interinjection } \\ \text { (time out) } \\ \text { lever presses }\end{array}\end{array}$

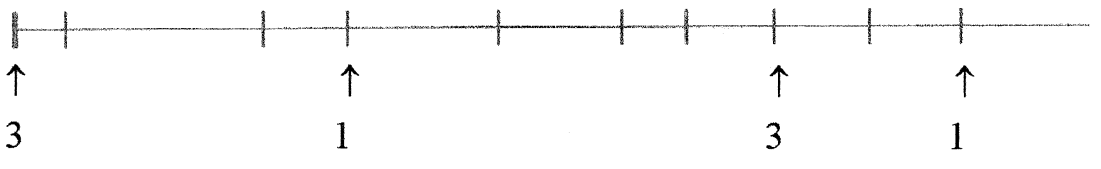

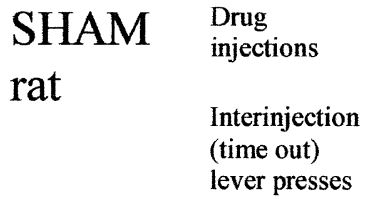

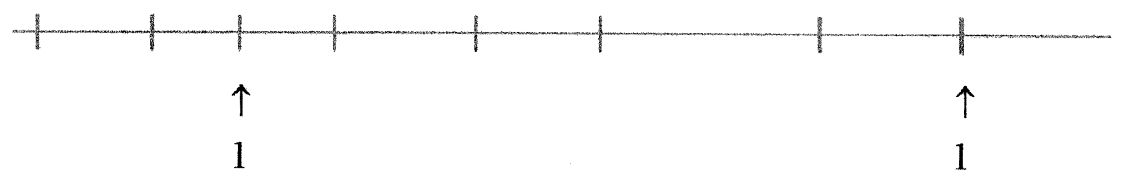

Figure 6. Baseline cocaine self-administration $(0.4 \mathrm{mg} / \mathrm{kg} /$ inj.) prior to extinction/reinstatement testing in NVHL and sham rats. A. NVHL rats continue to exhibit response perseveration at the active lever, with no difference in the number of injections taken compared with shams. B. Representative response records illustrating regularity in patterns of cocaine intake concomitant with response perseveration during the injection/time-out periods in an NVHL rat over a 30-min period compared with a sham animal. Hatch marks represent active lever responses that elicit a single drug injection. Arrows denote numbers of time-out responses in the 14-s time period after start of drug injection. Data in (A) are presented as the mean $\pm \mathrm{SEM},{ }^{*} p<.05$ by 2 -way ANOVA with repeated measures on test day.

$\left(\mathrm{F}_{1,17}=5.88, p<.05\right)$ and a Lesion $\mathrm{X}$ Dose interaction $\left(\mathrm{F}_{2,16}=3.97, p<.05\right)$. Separate analyses of each lever (Figure 7, panel C) found that NVHL rats responded more at the drug-paired lever than shams $\left(\mathrm{F}_{1,17}=4.4\right.$, $p=.05)$ following cocaine but not saline priming, resulting in a significant Lesion $X$ Dose interaction $\left(\mathrm{F}_{2,16}=\right.$
6.37, $p<.01)$. However, there were no effects of either Lesion or Dose on inactive lever responding ( $p$ values both $>$.10). One sham and two NVH lesioned animals were excluded from the analyses due to high ( $>30$ lever responses) in the 1-h period immediately preceding the reinstatement tests. 


\section{Extinction/Reinstatement of Cocaine Seeking SHAM (N=12), NVHL $(\mathrm{N}=10)$}
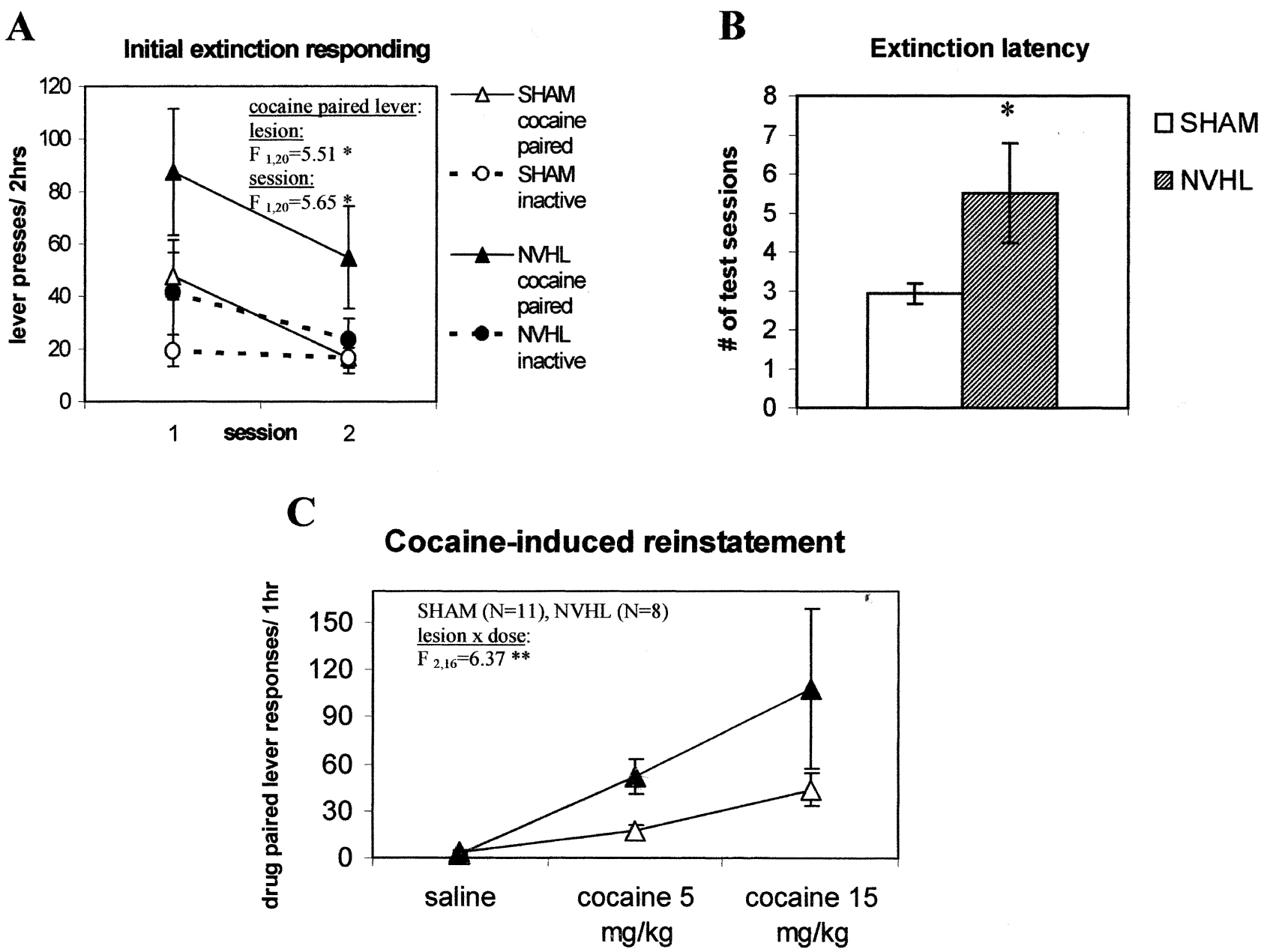

Figure 7. Drug-paired lever responding during extinction and reinstatement testing. A. NVHL rats show significantly higher rates of cocaine-paired, but not inactive, lever responses during first two extinction test sessions relative to shams. B. The latency to reach extinction is prolonged in NVHL rats criteria $(\leqslant 15$ responses on cocaine-paired lever, $\leqslant 30$ total on both levers in 2hr). C. Following achievement of extinction criteria, reinstatement induced by i.p. priming injections of cocaine is enhanced in NVHL relative to sham rats. Priming injections of saline or cocaine ( 5 and $15 \mathrm{mg} / \mathrm{kg}) \mathrm{were}$ delivered after $2 \mathrm{~h}$ of additional extinction conditions in the test chambers. Two NVHL and one sham animal were excluded from the analysis due to high baseline responding ( $>30$ total on both levers in second baseline hour). Data are presented as the mean $\pm \mathrm{SEM},{ }^{*} p<.05,{ }^{* *} p<.01$ by 2 -way ANOVA, or by 2 -tailed $t$-test.

\section{DISCUSSION}

NVHL rats exhibit alterations in behavior reinforced by both natural and drug rewards. First, they acquire sucrose self-administration more readily than sham-lesioned rats. This difference could not be directly attributed to increased spontaneous sampling of the levers, because spontaneous lever press responses were habituated to the same degree in all animals prior to acquisition testing. Similarly, it is unlikely that hunger or drive-related differences in the motivation for food rewards differed in NVHL rats, since both sham and NVHL rats showed similar latencies to consume the sucrose pellets once the lever-press task had been learned, and both groups had gained weight at similar rates prior to testing. It also is difficult to attribute these findings to the incentive motivational strength of sucrose rewards in NVHL rats, since the magnitude of sucrose-seeking responses in extinction testing, and the latency to achieve extinction criteria, failed to differ from shams. Previous studies have shown that these extinction measures primarily reflect the perceived reward value during prior training, rather than the motivational state of animals during extinction testing (Balleine and Dickinson 1998). 
Thus, facilitated acquisition of sucrose self-administration in NVHL rats may be more related to an enhanced capacity for instrumental learning, than to an increased sensitivity to the incentive value of sucrose rewards. However, it is also possible that acquisition was facilitated by heightened activity or arousal levels in the presence of sucrose rewards in NVHL rats relative to shams.

The possibility that instrumental learning is facilitated by NVHL is interesting given that similar lesions produce spatial learning deficits in a radial arm maze task that also utilized food rewards (Chambers et al. 1996). Spatial learning may be more dependent on dorsal rather than ventral hippocampal regions (Moser and Moser 1998; Vann et al. 2000), whereas instrumental learning involves stimulus-response associations that probably are more dependent on basal ganglia structures (Hollerman et al. 2000). One possibility is that reward-related learning involving ventral striatal mechanisms may be "disinhibited" by hippocampal lesions, as suggested previously (Burns et al. 1996; Chambers et al. 2001; Kelley and Mittleman 1999; Schmelzeis and Mittleman 1996). Another consideration is that stimulus-response contingencies learned during acquisition may utilize different neural substrates than those involved in extinction of these learned contingencies, as suggested for acquisition and extinction of Pavlovian associations (Lattal and Abel 2001).

Responding during acquisition of cocaine self-administration also was facilitated in NVHL rats relative to shams, despite the fact that sucrose reinforcement had been extinguished to similar response rates in both groups, and the active and inactive levers were reversed from the sucrose experiment. These differences primarily resulted from marked increases in "non-reinforced" response perseveration during each cocaine injection, and to a greater incidence of binge-like cocaine consumption, rather than to a consistent increase in the amount cocaine injections taken over the four weeks of acquisition testing. This effect may reflect either: (1) a greater capacity for instrumental learning as stated above; (2) an increase in sensitivity to cocaine reinforcement; or (3) cocaine-induced response perseveration in NVHL rats. However, in contrast to sucrose reinforcement, there was no significant effect of NVH 1 of NVHL rats had acquired cocaine self-administration after two weeks, whereas shams required three weeks to reach $50 \%$ acquisition. Moreover, there was no evidence for increased sensitivity to cocaine reinforcement in NVHL rats, since cocaine intake was similar at the lowest training dose during the first week of acquisition testing, and both groups showed ascending cocaine intake over the range of doses tested in subsequent weeks. Marginal increases in weekly cocaine intake at the higher training doses may reflect a slightly ac- celerated pace of acquisition, but did not translate into increased baseline cocaine intake following acquisition (Figure 6).

Such persistent perseveration of active lever responding during cocaine self-administration cannot be attributed to a generalized rate effect in NVHL rats, since the inactive lever was not increased relative to shams, and response rates during sucrose extinction failed to differ. Furthermore, response perseveration in NVHL rats continued even as the amount and pattern of cocaine self-administration stabilized prior to extinction/reinstatement testing. The greater incidence of binge-like self-administration throughout acquisition, coupled with an inability to inhibit redundant responding as self-administration patterns stabilized, is consistent with a failure to develop inhibitory feedback regulation leading to uncontrolled or impulsive cocaine seeking, and in some cases, cocaine taking behaviors in NVHL animals.

Another possibility is that $\mathrm{NVH}$ lesions produce a generalized rate-increasing effect during cocaine exposure. However, extinction tests were conducted in the absence of cocaine reinforcement, and yet cocaine-seeking responses at the drug-paired lever in NVHL rats were increased relative to shams, and required almost twice as many test sessions to reach extinction criteria. Given that response rates during extinction of sucrose reinforcement were identical in both groups, it is unlikely that higher response rates reflect impaired extinction learning mechanisms or other generalized rate effects, but rather, they reflect enhanced incentive motivation for cocaine after withdrawal. After about two weeks of withdrawal, and when animals had extinguished to similar low response levels (both in repeated extinction sessions and in the 1-h period immediately prior to reinstatement tests), experimenter-delivered cocaine injections induced markedly greater and selective responses at the drug-paired lever in NVHL animals relative to shams, whereas saline injections were without effect in both groups. Taken together, these results suggest that $\mathrm{NVH}$ lesions promote relapse to cocaine seeking, whether induced by exposure to the cocaine-associated context (extinction), or to a priming injection of cocaine (reinstatement).

Our previous studies suggest that vulnerability or propensity for reinstatement is closely associated with the higher levels of cocaine intake among individual animals (Sutton et al. 2000). In the present study, it is notable that total lifetime cocaine intake in NVHL rats (399.3 \pm $49.9 \mathrm{mg} / \mathrm{kg} / \mathrm{rat}$ ) failed to differ statistically from shams $(329.9 \pm 18.1 \mathrm{mg} / \mathrm{kg} / \mathrm{rat})$, although it is probable that an experimentally imposed limitation of 120 daily injections precluded a difference in this measure (see Figure 5, panel A). Moreover, neither the time from cocaine selfadministration to reinstatement testing (NVHL, $13.6 \pm$ 0.8 days; sham, $14.9 \pm 1.2$ days), nor the time from the 
last extinction test to reinstatement testing (NVHL, $6.6 \pm$ 1.1 days; shams $8.3 \pm 1.8$ days) differed statistically. Thus, marked increases in cocaine seeking in NVHL rats cannot readily be explained by differences in drug selfadministration history, per se. Instead, these results could reflect a direct influence of $\mathrm{NVH}$ lesions on the incentive motivational aspects of cocaine, independent from factors that regulate drug intake. In any event, response perseveration and uncontrolled binge-like selfadministration patterns are reminiscent of impulsivity, and are followed by marked increases in cocaine seeking responses weeks after cocaine self-administration. These changes parallel critical diagnostic features of the addicted phenotype in this animal model of schizophrenia (DSM-IV 1994). Furthermore, the differential ability of $\mathrm{NVH}$ lesions to modulate extinction from sucrose- and cocaine-reinforcement may suggest a preferential enhancement of neuroplastic processes underlying drug addiction, while having less impact on natural reinforcement substrates. Conversely, this preferential interaction could reflect the greater capacity for cocaine to activate natural reinforcement substrates relative to sucrose in NVHL rats.

Table 1 shows the behavioral profiles of sham and bilateral NVHL rats, along with five rats with unilateral NVHL. While the low number of unilateral NVHL rats precludes statistical analysis, certain behavioral effects are notable. Unilateral NVHL rats performed similar to shams in spontaneous lever responding, acquisition of sucrose and cocaine self-administration. However, in contrast to sham and bilateral lesions, extinction from sucrose is prolonged in unilateral NVHL rats, similar to the effects of both lesions on extinction from cocaine. These preliminary results suggest that NVHL-induced facilitation of instrumental learning may require extensive bilateral damage, whereas sparing contralateral hippocampal inputs generally disrupts extinction learning relative to bilateral lesions. In contrast, unilateral lesions of the CA1 region in the adult rats are reported to "disinhibit" the reinforcing effects of contralateral brain stimulation reward, but do not alter extinction of this behavior (Zimmerman et al. 1997). Although further study of these phenomena is warranted, it is possible that unilateral and bilateral NVH lesions produce differential developmental alterations in adults.

To our knowledge, the effects of adult-onset ventral hippocampal lesions in acquisition and extinction of cocaine self-administration have not been reported. However, adult rats subjected to bilateral ventral hippocampal lesions show resistance to extinction from food-reinforced behaviors (Clark et al. 1992), an effect that differs from neonatal hippocampal lesions in the present study. Adult hippocampal lesions also decrease food neophobia, and increase the reinforcing effects of sucrose and brain stimulation reward (Burns et al. 1996; Kelley and Mittleman 1999; Schmelzeis and Mittleman
1996), consistent with the effects of neonatal lesions on sucrose and cocaine reinforcement in the present study. In contrast, disruption of hippocampal theta waves of adult rats promotes extinction from brain stimulation reward (Holt and Gray 1983), suggesting that hippocampal activity is involved in bi-directional regulation of motivated behavior under reinforcement and extinction conditions. These studies, along with recent anatomical and physiological findings, have led to the hypothesis that the ventral hippocampus and efferent cortical structures are part of a complex neural network where glutamatergic inputs ultimately converge onto nucleus accumbens neurons to regulate goal-directed behavior elicited by dopaminergic inputs (Hollerman et al. 2000). Inadequate or disrupted hippocampal input to frontal cortical and ventral striatal regions may compromise this inhibitory regulation, resulting in perseveration of goal-directed (drug-seeking) behavior (Gurden et al. 1999; Mulder et al. 1998; Ridley 1994).

In schizophrenia, developmental abnormalities in ventral hippocampal and associated structures are hypothesized to disrupt this circuitry leading to motivational disturbances that increase vulnerability to addiction in schizophrenic patients who use drugs (Chambers et al. 2001). Consistent with this view, the NVHL model of developmental alterations in schizophrenia has been shown to produce numerous cognitive and behavioral abnormalities emergent after puberty similar to psychopathology in schizophrenia (see Introduction). Our results do not discern whether the effects of NVH lesions on drug addiction behaviors are generalizable to adult onset lesions, or are specific to developmental abnormalities. Lipska and colleagues have described differential effects on the developmental onset of expression of DAmediated behaviors in rats with ventral hippocampal lesions induced at various prepubertal ages and adulthood (Lipska et al. 1995b; Wood et al. 1997). Using prepulse inhibition of startle (PPI), Lipska and colleagues demonstrated that inherent basal deficits in PPI are evident post-pubertal, but not pre-pubertal, following NVHLs (Lipska et al. 1995c), consistent with the post-pubertal emergence of schizophrenia in humans. In contrast, Swerdlow and colleagues found that adult ventral hippocampal lesions produce only mild impairment in basal PPI that fully recovers after four weeks, whereas apomorphine-induced disruption of PPI remains enhanced similar to NVHL rats (Swerdlow et al. 2000). These findings support the idea that NVHL rats model certain developmental deficits that differ from the direct effects of adult lesions, that is, enduring inherent deficits in sensory gating processes, whereas sensitivity to psychostimulant-induced deficits endures in both cases. Our findings suggest that $\mathrm{NVH}$ lesions also promote instrumental behavior associated with both natural and psychostimulant rewards, consistent with increased addiction vulnerability in adulthood. Although it is not clear when these 
traits emerge, it is possible that adult lesions could produce similar enduring alterations, especially with respect to psychostimulant responses. However, a recent study found that ventral subicular lesions in adults moderately impair acquisition of cocaine self-administration (Caine et al. 2001).

The high degree of substance use disorders comorbid with schizophrenia has been suggested to reflect a variety of influences, including neuroleptic treatment, selfmedication, and social factors. Our results in NVHL rats suggest that substance abuse vulnerability could represent a premorbid state in schizophrenic patients, and that these patients are particularly prone to develop addictive behavior based on developmental abnormalities independent of these other possible influences. An alternative interpretation, based on self-medication hypotheses, would hold that NVHL rats show increased measures of cocaine self-administration in an attempt to alleviate other lesion-related symptoms. While our results support the idea that NVH lesions increase the incentive motivational effects of cocaine, the possibility that the lesions enhance the negative reinforcing properties of cocaine cannot be ruled out. However, this interpretation seems less plausible because NVHL-related symptoms such as deficits in working memory do not improve with psychostimulant administration, and psychostimulant-induced hyperlocomotion and sensory gating deficits are worsened rather than ameliorated (Lipska et al. 1995c; Lipska and Weinberger 1994; Chambers et al. 1996). Moreover, these animal studies parallel clinical reports of symptom exacerbation and increased psychiatric morbidity in schizophrenia patients with substance abuse (Buckley 1998; Dixon 1999). These findings, along with our results, would seem to support the idea that vulnerability to substance abuse and classic psychpathology are both primary disease symptoms in schizophrenia that stem from common developmental neuropathology in the ventral hippocampus and its impact on other limbic structures.

\section{ACKNOWLEDGMENTS}

This work was supported by a special Neuroscience Research Fellowship Training Grant provided by the Veterans Administration, A Junior Investigator Award Provided by the National Alliance for Research on Schizophrenia and Depression (NARSAD), and by United States Public Health Service Grant DA 10460. We wish to thank Ralitza Gueorguieva, Ph.D., for her statistical consultations.

\section{REFERENCES}

Balleine BW, Dickinson A (1998): Goal-directed instrumental action: contingency and incentive learning and their cortical substrates. Neuropharmacology 37:407-419
Becker A, Grecksch G (2000): Social memory is impaired in neonatally ibotenic acid lesioned rats. Behav Brain Res 109:137-140

Becker A, Grecksch G, Bernstein HG, Hollt V, Bogerts B (1999): Social behavior in rats lesioned with ibotenic acid in the hippocampus: quantitative and qualitative analysis. Psychopharmacology (Berl) 144:333-338

Benes FM, Beretta S (2001): GABAergic interneurons: implications for understanding schizophrenia and bipolar disorder. Neuropsychopharmacology 25:1-27

Bernstein HG, Grecksch G, Becker A, Hollt V, Bogerts B (1999): Cellular changes in rats brain areas associated with neonatal hippocampal damage. Neuroreport 10:2307-2311

Buckley PF (1998): Substance abuse in schizophrenia: a review. J Clin Psychiatry 59:26-30

Burns LH, Annett L, Everett BJ, Robbins TW, Kelley AE (1996): Effects of lesions to amygdala, ventral subiculum, medial prefrontal cortex, and nucleus accumbens on the reaction to novelty: implication for limbic-striatal interactions. Behav Neurosci 110:60-73

Caine SB, Humby T, Robbins TW, Everitt BJ (2001): Behavioral effects of psychomotor stimulants in rats with dorsal or ventral subiculum lesions: locomotion, cocaine self-administration, and prepulse inhibition of startle. Behav Neurosci 115:880-894

Chambers RA, Krystal JK, Self DW (2001): A neurobiological basis for substance abuse comorbidity in schizophrenia. Biol Psychiatry 50:71-83

Chambers RA, Moore J, McEvoy JP, Levin ED (1996): Cognitive effects of neonatal hippocampal lesions in a rat model of schizophrenia. Neuropsychopharmacology 15:587-594

Clark AJM, Feldon J, Rawlins JNP (1992): Aspiration lesions of rat ventral hippocampus disinhibit responding in conditioned suppression or extinction, but spare latent inhibition and the partial reinforcement extinction effect. Neuroscience 48:821-829

Crow TJ, Deakin JFW, Longden A (1977): The nucleus accumbens-possible site of action of antipsychotic action of neuroleptic drugs? Physiological Medicine 7:213-221

DeQuardo JR, Carpenter CF, Tandon R (1994): Patterns of substance abuse in schizophrenia: nature and significance. J Psychiatr Res 28:267-275

Dixon L (1999): Dual diagnosis of substance abuse in schizophrenia: prevalence and impact on outcomes. Schizophr Res 35:S93-S100

Floresco SB, Todd CL, Grace AA (2001): Glutamatergic afferents from the hippocampus to the nucleus accumbens regulate activity of ventral tegmental area dopamine neurons. J Neurosci 21:4915-4922

Grecksch G, Bernstein HG, Becker A, Hollt V, Bogerts B (1999): Disruption of latent inhibition in rats with postnatal hippocampal lesions. Neuropsychopharmacology 20:525-532

Groenewegen HJ, Wright CI, Uylings HBM (1997): The anatomical relationships of the prefrontal cortex with limbic structures and the basal ganglia. J Psychopharmacol 11:99-106

Gurden H, Tassin JP, Jay TM (1999): Integrity of mesocorti- 
cal dopaminergic system is necessary for complete expression of in vivo hippocampal-prefrontal cortex long-term potentiation. Neuroscience 94:1019-1027

Heckers S, Rauch SL, Goff D, Savage CR, Schacter DL, Fischman AJ, Alpert NM (1998): Impaired recruitment of the hippocampus during conscious recollection in schizophrenia. Nat Neurosci 1:318-323

Hollerman JR, Tremblay L, Schultz W (2000): Involvement of basal ganglia and orbitofrontal cortex in goal directed behavior. Progress in Brain Research 126:193-215

Holt L, Gray JA (1983): Proactive behavioral effects of the theta-blocking septal stimulation in the rat. Behav Neural Biol 39:7-21

Jay TM, Glowinski J, Thierry AM (1995): Inhibition of hippocampo-prefrontal cortex excitatory responses by the mesocortical DA system. Neuroreport 6:1845-1848

Kelley AE, Domesick VB (1982): The distribution of the projection from the hippocampal formation to the nucleus accumbens in the rat: an anterograde and retrogradehorseradish peroxidase study. Neuroscience 7:23212335

Kelley SP, Mittleman G (1999): Effects of hippocampal damage on reward threshold and response rate during selfstimulation of the ventral tegmental area in the rat. Behav Brain Res 99:133-141

Koob GF, Le Moal M (2001): Drug addiction, dysregulation of reward, and allostasis. Neuropsychopharmacology 24:97-129

Lattal KM, Abel T (2001): Different requirements for protein synthesis in acquisition and extinction of spatial preferences and context-evoked fear. J Neurosci 21:5773-5780

Lieberman JA, Kinon BJ, Loebel AD (1990): Dopaminergic mechanisms in idiopathic and drug- induced psychosis. Schizophr Bull 16:97-110

Lillrank SM, Lipska BK, Bachus SE, Wood GK, Weinberger DR (1996): Amphetamine-induced c-fos mRNA expression is altered in rats with neonatal ventral hippocampal damage. Synapse 23:292-301

Lillrank SM, Lipska BK, Kolachana BS (1999): Attenuated extracellular dopamine levels after stress and amphetamine in the nucleus accumbens of rats with neonatal ventral hippocampal damage. J Neural Transm 106: 183-196

Lipska BK, Chrapusta SJ, Egan MF, Weinberger DR (1995a): Neonatal excitotoxic ventral hippocampal damage alters dopamine response to mild repeated stress and to chronic haloperidol. Synapse 20:125-130

Lipska BK, Jaskiw GE, Braun AR, Weinberger DR (1995b): Prefrontal cortical and hippocampal modulation of haloperidol-induced catalepsy and apomorphine-induced stereotypic behaviors in the rat. Biol Psychiatry 38:255262

Lipska BK, Jaskiw GE, Weinberger DR (1993): Postpubertal emergence of hyperresponsiveness to stress and to amphetamine after neonatal excitotoxic hippocampal damage: a potential animal model of schizophrenia. Neuropsychopharmacology 9:67-75

Lipska BK, Swerdlo NR, Geyer MA, Jaskiw GE, Braff DL, Weinberger DR (1995c): Neonatal excitotoxic hippocampal damage in rats causes post-pubertal changes in prepulse inhibition of startle and its disruption by apo- morphine. Psychopharmacology (Berl) 122:35-43

Lipska BK, Weinberger DR (1994): Subchronic treatment with haloperidol and clozapone in rats with neonatal excitotoxic hippocampal damage. Neuropsychopharmacology 10:199-205

Luchins DJ (1992): Repetitive behaviors in chronically institutionalized schizophrenic patients. Schizophr Res 8:119-123

Moser MB, Moser EI (1998): Functional differentiation in the hippocampus. Hippocampus 8:608-619

Mulder AB, Hodenpijl MG, Lopez de Silva FH (1998): Electrophysiology of the hippocampal and amygdaloid projections to the nucleus accumbens of the rat: convergence, segregation, and interactions of inputs. J Neurosci 18:5095-5102

Nestler EJ, Aghajanian GK (1997): Molecular and cellular basis of addiction. Science 278:58-63

O'Donnell P, Greene J, Pabello N, Lewis BL, Grace AA (1999): Modulation of cell firing in the nucleus accumbens. Ann N Y Acad Sci 877:157-175

O'Donnell PO, Grace AA (1995): Synaptic interactions among excitatory afferents to the nucleus accumbens neurons: hippocampal gating of prefrontal cortical input. J Neurosci 15:3622-3639

Ridley RM (1994): The psychology of perseverative and stereotyped behavior. Prog Neurobiol 44:221-231

Robbins TW, Everitt BJ (1996): Neurobehavioral mechanisms of reward and motivation. Curr Opin Neurobiol $6: 228-236$

Robbins TW, Granon S, Muir JL, Durantou F, Harrison A, Everitt BJ (1998): Neural systems underlying arousal and attention. Implications for drug abuse. Ann N Y Acad Sci 846:222-237

Robinson TE, Kolb B (1999): Alterations in the morphology of dendrites and dendritic spines in the nucleus accumbens and prefrontal cortex following repeated treatment with amphetamine or cocaine. Eur J Neurosci 11:1598-1604

Sams-Dodd F, Lipska BK, Weinberger DR (1997): Neonatal lesions of the rat ventral hippocampus results in hyperlocomotion and deficits in social behavior in adulthood. Psychopharmacology (Berl) 132:303-310

Schmajuk NA, Cox L, Gray JA (2001): Nucleus accumbens, entorhinal cortex and latent inhibition: a neural network model. Behav Brain Res 118:123-141

Schmelzeis MC, Mittleman G (1996): The hippocampus and reward: effects of hippocampal lesions on progressiveratio responding. Behav Neurosci 110:1049-1066

Schroeder H, Grecksch G, Becker A, Bogerts B, Hoellt V (1999): Alterations of the dopaminergic and glutamatergic neurotransmission in adult rats with postnatal ibotenic acid hippocampal lesion. Psychopharmacology (Berl) 145:61-66

Self DW (1998): Neural substrates of drug craving and relapse in drug addiction. Ann Med 30:379-389

Sesack SR, Pickel VM (1990): In the rat medial nucleus accumbens, hippocampal and catecholaminergic terminal converge on spiny neurons and are in apposition to each other. Brain Res 527:266-279

Skopec M, Rosenberg SD, Tucker GJ (1976): Sexual behavior in schizophrenia. Med Aspects Hum Sex 10:32-47 
Sutton MA, Karanian DA, Self DW (2000): Factors that determine a propensity for cocaine-seeking behavior during abstinence in rats. Neuropsychopharmacology 22:626-641

Swerdlow NR, Taaid N, Halim N, Randolph E, Kim Y, Auerbach P (2000): Hippocampal lesions enhance the startle gating-disruptive effects of apomorphine in rats: a parametric assessment. Neuroscience 96:523-536

Vann SD, Brown MW, Erichsen JT, Aggleton JP (2000): Fos imaging reveals differential patterns of hippocampal and parahippocampal subfield activation in rats in respnse to different spatial memory tests. J Neurosci 20:2711-2718

Verwer RVH, Meijer RJ, Van Uum HFM, Witter MP (1997): Collateral projections from the rat hippocampal formation to the lateral and medial prefrontal cortex. Hippocampus 7:397-402

Weinberger DR (1999): Cell biology of the hippocampal formation in schizophrenia. Biol Psychiatry 45:395-402
Weinberger DR, Aloa MS, Goldberg TE, Berman KF (1994): The frontal lobes and schizophrenia. J Neuropsychiat 6: $419-427$

White FJ, Kalivas PW (1998): Neuroadaptations involved in amphetamine and cocaine addiction. Drug Alcohol Depend 51:141-153

Wise RA (1998): Drug-activation of brain reward pathways. Drug Alcohol Depend 51:13-22

Wood GK, Lipska BK, Weinberger DR (1997): Behavioral changes in rats with early ventral hippocampal damage vary with age at damage. Brain Res Dev Brain Res 101: $17-25$

Zimmerman PK, Wagner U, Krauth J, Huston JP (1997): Unilateral lesion of dorsal hippocampus enhances reinforcing lateral hypothalamic stimulation in the contralateral hemisphere. Brain Res Bull 44:265-271 